\title{
Detection of Calprotectin and Apoptotic Activity in the Colon of Marmosets with Chronic Diarrhea
}

\author{
Erika NAKASHIMA ${ }^{1)}$, Yasushi OKANO ${ }^{1)}$, Kimie NIIMI ${ }^{1)}$ and Eiki TAKAHASHI ${ }^{1) *}$ \\ 1) Research Resources Center, RIKEN Brain Science Institute, 2-1 Hirosawa, Wako, Saitama 351-0198, Japan
}

(Received 20 May 2013/Accepted 10 July 2013/Published online in J-STAGE 24 July 2013)

\begin{abstract}
The common marmoset (Callithrix jacchus) is used as a non-human primate laboratory animal. Marmoset wasting syndrome (MWS) is a disease endemic to captive colonies, and the pathogenesis is unclear. In the present study, marmosets with chronic bloody highviscosity diarrhea, which is a contributing factor to MWS, were evaluated, and inflammation in the colon was found. Calprotectin is a surrogate marker of intestinal inflammation and induces apoptosis. Marmosets with chronic diarrhea exhibited higher levels of fecal calprotectin. Histochemical analyses showed high expression of calprotectin in the extravascular neutrophils and apoptosis in the chronic colitis lesions. No internal microbiological diseases were identified. Although the cause of chronic colitis was not identified, the marmoset could be a useful model of inflammatory bowel disease.
\end{abstract}

KEY WORDS: apoptosis, calprotectin, colitis, diarrhea, marmoset.

doi: 10.1292/jvms.13-0257; J. Vet. Med. Sci. 75(12): 1633-1636, 2013

The common marmoset (Callithrix jacchus) is a small New World primate that is native to eastern Brazil and has been used in biomedical research in areas, such as neuroscience, reproductive biology, infectious disease and drug development [6]. Compared to Macaca species, the common marmoset has several advantages, such as a small body size, availability, easy breeding in captivity, low husbandry cost, avoidable zoonotic issues, early sexual maturation and a short gestation period $[1,11]$. Thus, the number of studies using common marmosets as a non-human primate laboratory animal has increased in recent years [6, 9]. However, marmoset wasting syndrome (MWS), which includes chronic diarrhea, decreased muscle mass and weight loss, is a disease endemic to captive colonies of common marmosets [5, 13]. Although numerous studies have been conducted on the nutritional and infectious factors, the pathogenesis of this life-threatening disease remains unclear. In a previous study, chronic colitis was considered one of the most important contributing factors for the development of MWS [5].

Fecal calprotectin assays have been used to predict intestinal inflammation among patients with chronic diarrhea [3]. Calprotectin, which is a combination of the $8 \mathrm{kDa} \mathrm{S} 100 \mathrm{~A} 8$ (also called Calgranulin A or myeloid related protein 8, MRP8) and the $14 \mathrm{kDa}$ S100A9 (also called Calgranulin B or myeloid related protein 14, MRP14), is a member of the $\mathrm{S} 100$ protein family, a group of calcium-binding molecules with an important role in immunity modulation. Calprotectin is a major protein in neutrophilic granulocytes, which accounts for $60 \%$ of the total proteins in the cytosol fraction [8], and

*Correspondence to: Takahashi, E., Research Resources Center, RIKEN Brain Science Institute, 2-1 Hirosawa, Wako, Saitama 351-0198, Japan.

e-mail: etakahashi@brain.riken.jp

(C)2013 The Japanese Society of Veterinary Science its presence in feces is directly proportional to inflammatory neutrophil migration towards the intestinal tract $[3,15]$. Calprotectin induces secretions of various chemokines and promotes apoptosis $[3,15]$.

Recently, marmosets in our colony showed clinical signs similar to marmosets with MWS, especially chronic diarrhea. As a first step toward understanding the mechanisms of chronic diarrhea, we investigated the presence of inflammation, calprotectin and apoptosis in the colon of marmosets with chronic diarrhea.

The Animal Experiments Committee of RIKEN (Wako, Japan) approved the animal procedures, and the animals were handled in accordance with the Institutional Guidelines for Experiments using Animals. Common marmosets (Callithrix jacchus) from a breeding colony at the RIKEN Brain Science Institute (Wako, Japan) were maintained at $27^{\circ} \mathrm{C}$ and $50 \%$ humidity on a 12 -hr light-dark cycle. Chronic diarrhea was defined as persistent high-viscosity diarrhea for more than 2 weeks. A control group consisted of marmosets without diarrhea for more than 2 months. The marmosets had no external injury or chronic vomiting and were free of the following microorganisms: Cytomegalovirus, Shigella spp., Salmonella enteritidis, Vibrio parahaemolyticus, Campylobacter jejuni, Campylobacter coli, Clostridium difficile, Clostridium perfringens, Mycobacterium tuberculosis, Helicobacter pylori, Chlamydia trachomatis, Chlamydophila pneumonia and Entamoeba histolytica. These microorganisms were measured in the clinical laboratory of the Health Sciences Research Institute (Yokohama, Japan). Diarrheagenic Escherichia coli including enteropathogenic, enteroinvasive, enterotoxigenic, enterohemorrhagic and enteroaggregative strains was examined using PowerChek ${ }^{\mathrm{TM}}$ diarrheal E. coli Detection Kits (Kogene Biotech Co., Ltd., Seoul, Korea). We performed fecal occult blood tests using Quick Chaser (MIZUHO MEDY Co., Ltd., Saga, Japan) to detect subtle blood loss in the intestinal tract. To conduct 
Table 1. Result of biological markers for each marmoset

\begin{tabular}{lcccccccccc}
\hline & Sex & $\begin{array}{c}\text { Age } \\
(\text { months })\end{array}$ & $\begin{array}{c}\text { Bloody } \\
\text { fecal }\end{array}$ & $\begin{array}{c}\text { Hemoglobin } \\
(\mathrm{g} / \mathrm{d} l)\end{array}$ & $\begin{array}{c}\text { Hematocrit } \\
(\%)\end{array}$ & $\begin{array}{c}\text { RBCs } \\
\left(\times 10^{6} / \mu l\right)\end{array}$ & $\begin{array}{c}\text { Calprotectin } \\
(\mathrm{ng} / \mathrm{m} l)\end{array}$ & $\begin{array}{c}\text { WBCs } \\
\left(\times 10^{3} / \mu l\right)\end{array}$ & $\begin{array}{c}\text { Platelets } \\
\left(\times 10^{5} / \mu l\right)\end{array}$ & $\begin{array}{c}\text { Albumin } \\
(\mathrm{g} / \mathrm{d} l)\end{array}$ \\
\hline CD 1 & Male & 72 & Positive & 5.1 & 17.3 & 2.6 & 315.7 & 9.1 & 3.5 & 2.0 \\
CD 2 & Female & 46 & Positive & 4.6 & 15.5 & 2.3 & 304.3 & 9.8 & 4.3 & 2.4 \\
CD 3 & Female & 73 & Positive & 5.5 & 17.4 & 2.7 & 226.1 & 8.9 & 4.4 & 2.0 \\
CD 4 & Female & 66 & Positive & 4.8 & 15.5 & 2.2 & 380.7 & 9.5 & 2.4 & 2.6 \\
\hline Control 1 & Male & 58 & Negative & 13.3 & 41.1 & 5.4 & 30.0 & 8.1 & 3.4 & 2.4 \\
Control 2 & Male & 40 & Negative & 13.2 & 40.1 & 5.4 & 33.6 & 8.0 & 3.2 & 2.1 \\
Control 3 & Male & 42 & Negative & 12.9 & 39.9 & 4.9 & 12.8 & 7.5 & 3.6 & 2.3 \\
Control 4 & Female & 51 & Negative & 14.2 & 42.2 & 5.2 & 24.8 & 8.2 & 3.4 & 1.8 \\
Control 5 & Female & 66 & Negative & 12.2 & 37.1 & 4.8 & 12.6 & 7.6 & 4.3 & 2.4 \\
Control 6 & Female & 43 & Negative & 14.1 & 42.2 & 5.6 & 16.4 & 8.0 & 3.7 & 2.5 \\
\hline
\end{tabular}

blood biochemical tests, blood was collected through the femoral vein using heparin-treated syringes. Red blood cells (RBCs), hematocrit, white blood cells (WBCs) and platelets were counted using the Celltac $\alpha$ (MEK-6450, NIHON KOHDEN, Tokyo, Japan). To purify plasma, blood was centrifuged at $1,800 \times g$ for $15 \mathrm{~min}$ within $1 \mathrm{hr}$ of blood collection. Plasma hemoglobin and albumin were measured using the Drychem 4000 system (FUJIFILM, Tokyo, Japan). Fecal calprotectin level was analyzed using PhiCal ${ }^{\mathbb{B}}$ Calprotectin ELISA Kit (Immundiagnostik AG, Bensheim, Germany). Data were analyzed using Student's $t$-tests (Excel Statistics 2006, SSRI, Tokyo, Japan) and are presented as means \pm standard error of the mean (SEM). Results were considered significant at $5 \%$ or less probability of error. Marmosets were anesthetized with an overdose of sodium pentobarbital and perfused with saline followed by $4 \%$ paraformaldehyde. Each marmoset colon was dissected after perfusion, fixed with Tissue Fixative (Gonostaff, Co., Ltd., Tokyo, Japan), then embedded in paraffin and sectioned at $6 \mu \mathrm{m}$ for hematoxylin and eosin (HE) staining, immunocytochemistry and terminal deoxynucleotidyl transferase-mediated dUTP nick-end labeling (TUNEL) assay. The protocols for immunocytochemistry [4] and TUNEL assay [12] were conducted as previously reported. To examine calprotectin expression with immunocytochemistry, a primary antibody to calprotectin (ab92331, Abcam plc., Cambridge, MA, U.S.A.) was used at a dilution of 1:750, and the secondary antibody (Polyclonal goat anti-rabbit immunoglobulins/biotinylated, E0432, Dako, Glostrup, Denmark) was used at a dilution of 1:600. TUNEL staining was performed using in situ Apoptosis Detection Kit (MK500, TAKARA BIO INC, Tokyo, Japan). The sections were counterstained with Mayer's Hematoxylin (Muto Pure Chemicals Co., Ltd., Tokyo, Japan), dehydrated and then mounted with Malinol (Muto Pure Chemicals Co., Ltd.).

Four animals (one male and three females) aged between 46 and 73 months were included in the chronic diarrhea (CD) group. Six animals (three males and three females) aged between 40 and 66 months were included in the control group. Diarrheagenic Escherichia coli was not detected in either group (data not shown). Data regarding biological markers for each animal are listed in Table 1. Although controls did not produce bloody feces, the $\mathrm{CD}$ group had bloody diarrhea in the fecal occult blood test. The biochemical markers were significantly different among groups in hemoglobin $(\mathrm{g} / \mathrm{d} /)$ (CD and control: $5.0 \pm 0.20$ and $13.3 \pm 0.31$, respectively; $P<0.01)$, hematocrit (\%) (CD and control: $16.4 \pm 0.53$ and $40.4 \pm 0.78$, respectively; $P<0.01), \mathrm{RBCs}\left(\times 10^{6} / \mu l\right)(\mathrm{CD}$ and control: $2.5 \pm 0.12$ and $5.2 \pm 0.13$, respectively; $P<0.01$ ), calprotectin $(n \mathrm{~g} / \mathrm{m} l)(\mathrm{CD}$ and control: $306.7 \pm 31.70$ and $21.7 \pm 3.70$, respectively; $P<0.01)$ and $\mathrm{WBCs}\left(\times 10^{3} / \mu l\right)$ (CD and control: $9.3 \pm 0.20$ and $7.9 \pm 0.12$, respectively; $P<0.05)$. There were not significant differences between groups in platelets $\left(\times 10^{5} / \mu l\right)(\mathrm{CD}$ and control: $3.7 \pm 0.46$ and 3.6 \pm 0.16 , respectively; $P>0.05)$ and albumin $(\mathrm{g} / \mathrm{d} l)(\mathrm{CD}$ and control: $2.3 \pm 0.15$ and $2.3 \pm 0.11$, respectively; $P>0.05$ ). To investigate the presence of inflammation, expression of calprotectin and activation of apoptosis, we analyzed four diarrhea and six control tissue specimens via histopathological tests including HE staining, immunocytochemistry and TUNEL assay. There were no polyps or cancer in the colon in any of the animals (data not shown). The colon specimens from the $\mathrm{CD}$ group revealed epithelial damage and disruption of crypt architecture (Fig. 1). Signs of colonic inflammation, including neutrophilic infiltration in the lamina propria of the mucosa, were evident in the colonic tissues of marmosets in the $\mathrm{CD}$ group. In the immunocytochemistry analysis, colon specimens from the CD group had increased numbers of specifically labeled calprotectin-positive cells in the lamina propria with respect to controls (Fig. 2A). TUNEL assay revealed apoptosis induction in all colitis specimens compared to controls (Fig. 2B).

MWS is one of the most important and least understood problems in laboratory-bred marmosets. Because the most common chronic symptom is diarrhea and pathological change is colitis [5], we focused on examining the colon of marmosets with chronic diarrhea using biochemical marker assays and histochemical examinations.

Fecal occult blood tests showed bloody, high-viscosity stools in the CD group, suggesting subtle blood loss in the intestinal tract. The levels of hemoglobin and hematocrit were lower in the $\mathrm{CD}$ group than in controls. The number of RBCs was also lower in the $\mathrm{CD}$ group. These results indicate anemia in the CD group caused by blood loss in the intestine. 


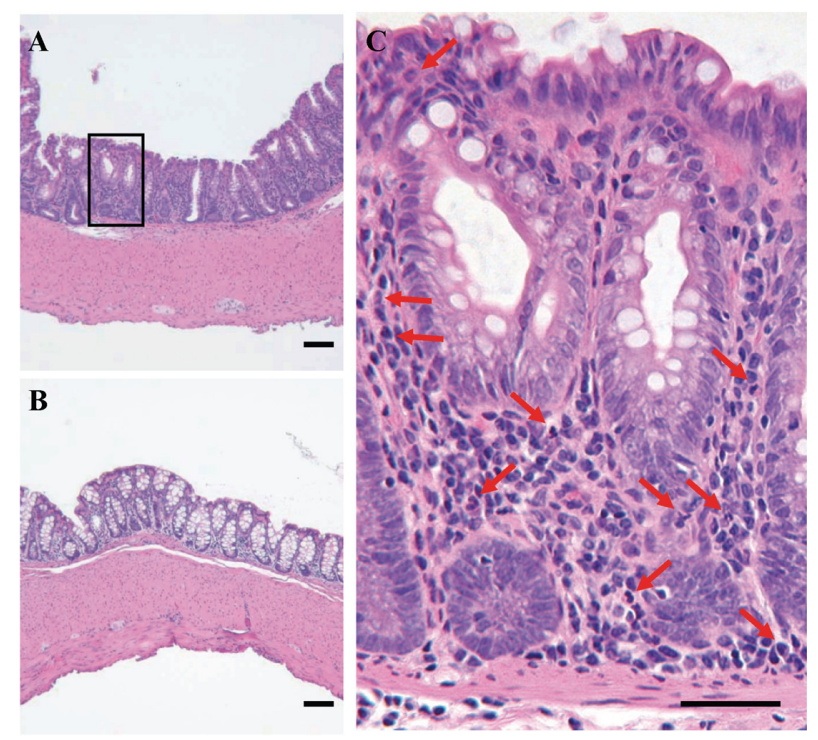

Fig. 1. Representative photomicrographs of sections of the colon in marmosets. HE staining showed the presence of inflammation in the colon of marmosets with chronic diarrhea (CD) group (A) compared with control (B). Scale bar, $100 \mu \mathrm{m}$. Magnification of A showed the migration of neutrophils (red arrows) in the lamina propria of the mucosa of CD group (C). Scale bar, $50 \mu \mathrm{m}$.
Conversely, platelet number was unaffected. Fecal calprotectin has been used as a non-invasive surrogate marker of intestinal inflammation diseases, such as inflammatory bowel disease [7]. Fecal calprotectin tests showed higher calprotectin concentrations in the CD group than in controls. To confirm the presence of inflammation, other biochemical marker was used. The number of WBCs was higher in the $\mathrm{CD}$ group than in controls, indicating that an inflammatory response occurred. No significant changes were observed in albumin parameters between $\mathrm{CD}$ and control groups, as previously reported [5]. Albumin concentration is frequently used to define the state of health, especially nutritional status [14]. However, our results indicate that albumin is not a suitable marker for the identification of chronic diarrhea. Reportedly, inflammatory diarrhea is caused by viral, bacterial or parasitic infections as well as by polyps, cancer or immune problems [7]. In the present study, microbiological tests showed negative results in both the CD and control groups. Anatomical findings showed no polyps or cancer in the colon of either group. These results indicate that inflammatory diarrhea is caused by aberrant activation of the colonic immune system. In HE staining, one of the main pathological features was infiltration of neutrophils in the colonic tissues of the $\mathrm{CD}$ group. In inflammatory reactions, calprotectin is detectable in elevated amounts that correlate to elevated levels of
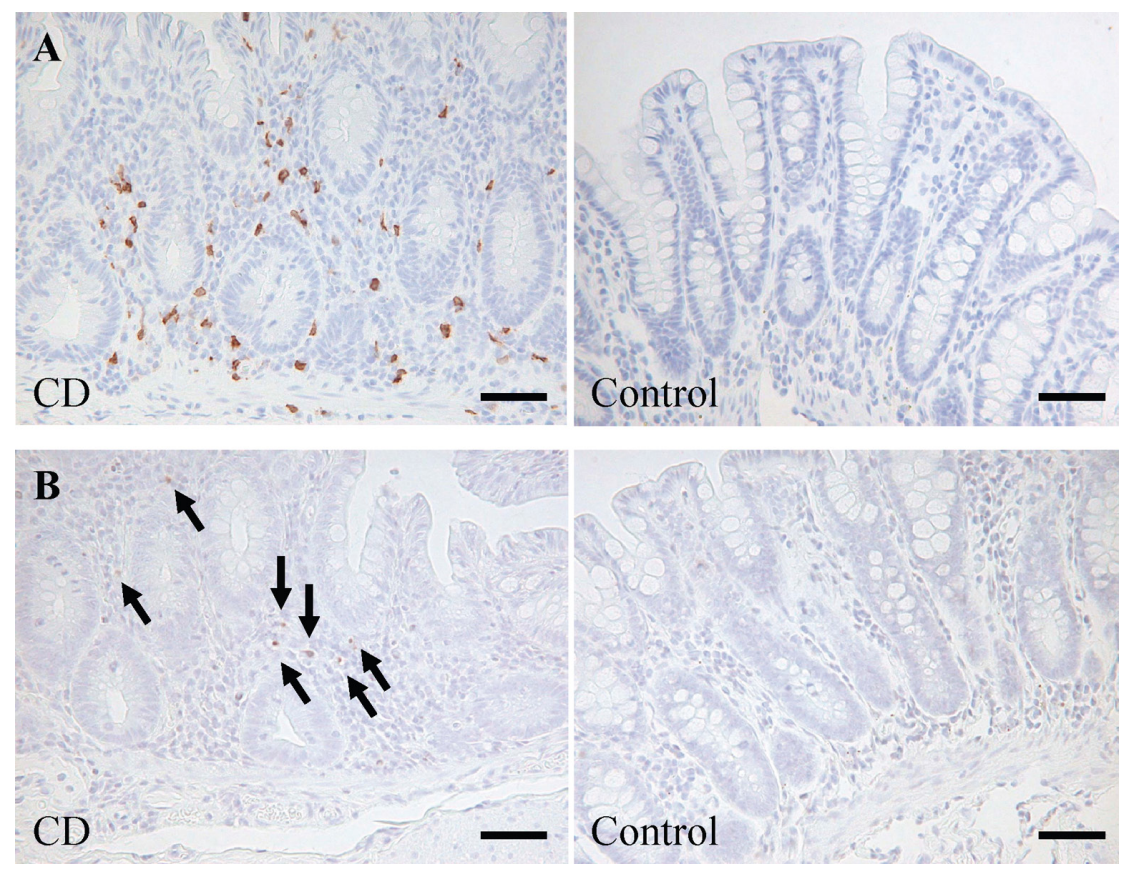

Fig. 2. Representative photomicrographs of calprotectin and apoptosis induction in the mucosa of colon in marmosets. Correlation between calprotectin expression and severity of inflammation was detected in the lamina propria of CD group (A, left panel) compared with controls (A, right panel). Scale bar, $50 \mu \mathrm{m}$. TUNEL assay revealed apoptosis induction in the lamina propria of CD group (B, left panel) compared with control (B, right panel). The apoptotic cells were indicated by black arrows. Scale bar, $50 \mu \mathrm{m}$. 
inflammatory neutrophils [3]. We examined the calprotectin expression pattern in colitis tissues in the CD group using immunocytochemistry. The $\mathrm{CD}$ group exhibited calprotectin expression in the extravascular cells. Conversely, there is little extravascular calprotectin-positive cell in the control group. These results indicate that calprotectin detected in the $\mathrm{CD}$ group feces was derived from inflammatory neutrophils in the colon.

Calprotectin has the capacity to induce apoptosis in various cells [3]. We performed a TUNEL assay to detect fragmented DNA associated with apoptosis. Although apoptotic cell death has been implicated as a major homeostatic mechanism of intestinal epithelium [2], we found that significantly increased apoptosis was correlated with the severity of colitis. These results suggest that calprotectin functions as a critical mediator of apoptosis and plays a central role in diarrhea.

The colon specimens from the CD group revealed the disruption of crypt architecture. In the present study, we could not clarify whether the loss of stem cells occurs nor not in the crypt of CD group. Although the effect of inflammatory chemokines on stem cells has been unclear, our data suggest that the damage with inflammation and apoptosis might induce abnormal number and differentiation of stem cells. It might be possible that inflammatory chemokines induced by calprotectin affect homeostatic mechanism of the colonic epithelium.

This study is the first report to show that marmosets with chronic diarrhea have a higher calprotectin expression and apoptosis in the inflammatory colon. Because calprotectin induces various inflammatory chemokines [3], which cause decreased muscle mass [10], chronic inflammation may be a primary insult in a majority of marmosets with MWS, and the release of proinflammatory cytokines secondarily causes decreased muscle mass and other syndrome manifestations. We will examine whether other inflammation indicators of marmosets with chronic diarrhea are larger than those of controls in the biochemical tests, since their comparisons between marmosets with chronic diarrhea and controls give more insight in the inflammatory background. Although further studies are needed to characterize colonic inflammation of marmosets with chronic diarrhea, our results suggest that fecal calprotectin can be a useful marker for detecting and monitoring colonic inflammation in marmosets with chronic diarrhea and that marmosets with chronic colitis could be a model of inflammatory bowel diseases.

ACKNOWLEDGMENTS. The authors would like to thank Yuka Hirayama, Kenji Nishio, Ayaka Oguchi, Miku Ito and Michiko Kamioka for maintaining marmoset colony and analyzing biochemical markers.

\section{REFERENCES}

1. Abbott, D. H., Barnett, D. K., Colman, R. J., Yamamoto, M. E. and Schultz-Darken, N. J. 2003. Aspects of common marmoset basic biology and life history important for biomedical research. Comp. Med. 53: 339-350. [Medline]

2. Edelblum, K. L., Yan, F., Yamaoka, T. and Polk, D. B. 2006. Regulation of apoptosis during homeostasis and disease in the intestinal epithelium. Inflamm. Bowel Dis. 12: 413-424. [Medline] [CrossRef]

3. Foell, D., Wittkowski, H. and Roth, J. 2009. Monitoring disease activity by stool analyses: from occult blood to molecular markers of intestinal inflammation and damage. Gut 58: 859-868. [Medline] [CrossRef]

4. Iriyama, T., Takeda, K., Nakamura, H., Morimoto, Y., Kuroiwa, T., Mizukami, J., Umeda, T., Noguchi, T., Naguro, I., Nishitoh, H., Saegusa, K., Tobiume, K., Homma, T., Shimada, Y., Tsuda, H., Aiko, S., Imoto, I., Inazawa, J., Chida, K., Kamei, Y., Kozuma, S., Taketani, Y., Matsuzawa, A. and Ichijo, H. 2009. ASK1 and ASK2 differentially regulate the counteracting roles of apoptosis and inflammation in tumorigenesis. EMBO J. 28: 843-853. [Medline] [CrossRef]

5. Logan, A. C. and Khan, K. N. 1996. Clinical pathologic changes in two marmosets with wasting syndrome. Toxicol. Pathol. 24: 707-709. [Medline] [CrossRef]

6. Mansfield, K. 2003. Marmoset models commonly used in biomedical research. Comp. Med. 53: 383-392. [Medline]

7. Payne, C. M., Fass, R., Bernstein, H., Giron, J., Bernstein, C., Dvorak, K. and Garewal, H. 2006. Pathogenesis of diarrhea in the adult: diagnostic challenges and life-threatening conditions. Eur. J. Gastroenterol. Hepatol. 18: 1047-1051. [Medline] [CrossRef]

8. Poullis, A., Foster, R., Northfield, T. C. and Mendall, M. A. 2002. Review article: faecal markers in the assessment of activity in inflammatory bowel disease. Aliment. Pharmacol. Ther. 16: 675-681. [Medline] [CrossRef]

9. Remington, E. D., Osmanski, M. S. and Wang, X. 2012. An operant conditioning method for studying auditory behaviors in marmoset monkeys. PLoS One 7: e47895. [Medline] [CrossRef]

10. Späte, U. and Schulze, P. C. 2004. Proinflammatory cytokines and skeletal muscle. Curr. Opin. Clin. Nutr. Metab. Care 7: 265-269. [Medline] [CrossRef]

11. Tardif, S. D., Smucny, D. A., Abbott, D. H., Mansfield, K., Schultz-Darken, N. and Yamamoto, M. E. 2003. Reproduction in captive common marmosets (Callithrix jacchus). Comp. Med. 53: 364-368. [Medline]

12. Tomizuka, K., Horikoshi, K., Kitada, R., Sugawara, Y., Iba, Y., Kojima, A., Yoshitome, A., Yamawaki, K., Amagai, M., Inoue, A., Oshima, T. and Kakitani, M. 2008. R-spondin1 plays an essential role in ovarian development through positively regulating Wnt-4 signaling. Hum. Mol. Genet. 17: 1278-1291. [Medline] [CrossRef]

13. Tucker, M. J. 1984. A survey of the pathology of marmosets (Callithrix jacchus) under experiment. Lab. Anim. 18: 351-358. [Medline] [CrossRef]

14. Wilkins, T., Jarvis, K. and Patel, J. 2011. Diagnosis and management of Crohn's disease. Am. Fam. Physician 84: 1365-1375. [Medline]

15. Yui, S., Nakatani, Y. and Mikami, M. 2003. Calprotectin (S100A8/S100A9), an inflammatory protein complex from neutrophils with a broad apoptosis-inducing activity. Biol. Pharm. Bull. 26: 753-760. [Medline] [CrossRef] 